Correspondents are asked to be brief

Continued Oral Therapy in Diabetes

H. S. Brodribb, D.M............... 108

Chloroquine-resistant Malaria?

L. J. Bruce-Chwatt, M.D., and J. M. D.

Roberts, D.P.H....................... 108

Endocrine and Metabolic Manifestations of Cancer

J. G. Azzopardi, M.D.; W. M. Keynes, F.R.C.s.109

Diagnosis of Potassium Chloride Stricture

R. J. Heald, F.R.C.S. . . . . . . . . . . . 109

Treatment of Asthma with Beclomethasone Aerosol

I. Grègg, B.M.; I. W. B. Grant, F.R.C.P.ED.,

and others................... 110

The Doctor in Conflict

P. Horgan, M.B.; F. Difford, M.B. . . . . . 111

"Sorcerers' Apprentices"

D. Gullick, M.B..............111
Payment by Colour

R. Hoffenberg, M.D.............111

Contaminated Drip Fluid

P. G. T. Ford, M.B..............111

Interstitial Cystitis

P. H. L. Worth, F.R.C.S., and R. T. Turner-

Warwick, F.R.C.S.; D. M. Essenhigh, F.R.C.S. 111

Papillary Necrosis in a Transplanted Kidney

C. Leroux-Robert, M.D., and others.......112

Suction Retractor



Islets of Munchausen

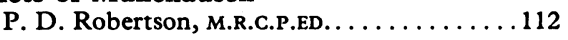

Bone Disease after Gastrectomy

W. M. Jordan, B.M. . . . . . . . . . . 113

Removal of Extruded I.U.D.

Ursula E. Mountrose, M.R.C.o.G., and W. L.

Whitehouse, F.R.C.S.ED............113
Recurrent Urinary Infections

H. G. Jones, M.R.C.S., D.M.R. . . . . . . 113

Simple Method of Measuring Disuse

Atrophy

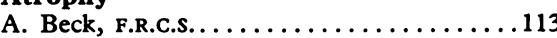

Foreign Body in the Appendix

T. G. Geddes, M.B., and others . . . . . . . 114

Squelching Caecum in Acute Appendicitis

S. N. Joffe, M.B., and J. H. Louw, F.R.C.S...114

Large Doses of Fluphenazine Enanthate

F. N. I. Fawzy, M.B., and others. . . . . . 114

Stereophonic Auscultation

B. L. P. Dalton, M.B...............115

Depressive Illness and Aggression in Belfast

D. Walsh, D.P.M.............. 115

Registration and the G.M.C.



\section{Continued Oral Therapy in Diabetes}

SIR,-I was very interested in Drs. A. M. Tomkins and Arnold Bloom's (11 March, p. 649) remarks on their findings. For many years it has been customary in the Hastings Diabetic Clinic to give many patients for whom oral treatment was necessary the right to vary their dose within narrow limits according to their urine record.

The vast majority were on chlorpropamide (464 in 1971) and it was found that even in those who initially required large doses a considerable reduction could be made automatically, an important point in avoiding hypoglycaemia in the elderly. They were instructed to reduce their dose by $100 \mathrm{mg}$ tentatively, if all the urine tests were free of sugar for three or four consecutive days. Should glycosuria return, the dose was restored, or even temporarily increased in times of stress or illness. This seems logical, when one considers how successfully patients vary their insulin according to tests-a much more potent treatment.

Special permission is given to start the first break with the final $100 \mathrm{mg}$. In quite a high proportion of cases, including those

\section{Chloroquine-resistant Malaria?}

SIR,-A report has been published in them were from the Embu area in central Arzneimittel Forschung (Drug Research) Kenya, north of Kitui. The authors state 1970, 29, No. 9, pp. 1206-1210 under the that "the majority of these patients were authorship of T. Yoshinaga, Y. Tsutsumi, K. suffering from tropical malaria ( $P$. falciTsunoda, and $Y$. Yamamura under the title parum) and $15 \%$ of them were suffering "Studies on the Antimalarial Property of from a mixed infection of $P$. falciparum and 4-methoxy-6 Sulfanil Amidopytimidine $P$. vivax." "These patients were treated in Monohydrate (Sulfamonomethoxine: DJ two groups, one of them by intravenous 1550) and on its Effect on the Chloroquine- route, the other orally; $40 \mathrm{mg} / \mathrm{kg}$ of sulresistant Malaria." This paper describes a famonomethoxine was injected twice a day study carried out on 279 African patients for two days and $20 \mathrm{mg} / \mathrm{kg}$ of this drug was from four areas in Kenya, but most (193) of administered orally on the first day, and
$10 \mathrm{mg} / \mathrm{kg}$ on the second to fourth days."

The authors present nine graphs showing parasitaemia of 20 patients, of whom 17 were treated by various dosages of the tested compound and three were treated by intramuscular injections or oral administration of chloroquine at $20-40 \mathrm{mg} / \mathrm{kg}$ for four to six days. The amount of chloroquine in terms of base was not stated.

The graphs indicate that the three adult patients treated with chloroquine had a parasitaemia of the order of $10^{3}$ parasites per $10^{4}$ erythrocytes; in other words that about $10 \%$ of their red blood cells were parasitized. Such parasitaemia indicates an extremely severe infection calling for emergency treatment. There is no evidence that such treatment was given. The authors report that "the numbers of malaria parasites did not decrease during this treatment" by chloroquine, but that the effect of sulfamonomethoxine on parasitaemia was rapid in almost all observed cases.

The authors then add that "although the studies ... . are not completed our studies ... show the definite existence of chloroquine resistant malaria" and that "sulfamonomethoxine is effective not only on chloroquine-sensitive malaria, but also on chloroquine-resistant malaria in Kenya."

This blunt statement is of such importance that one is justified in looking carefully at the methods employed in this drug trial. Unfortunately it appears that the main method of blood examination used by the authors was unconventional, to say the least. It consisted first of taking $1 \mathrm{ml}$ of the patient's blood, haemolysing it in $0.3 \%$ saponine solution; then " 10,000 ghost erythrocytes in the sediment of this material obtained after centrifugation were examined with phase microscope and the number of ghost erythrocytes which contained malaria parasites was shown as a percentage." 
If only because of this unusual method of examination many statements of this paper must not be accepted without reservations. And yet the publication of this report has already created much concern. It is likely that the authors did not fully realize the significance of a field study claiming to find the evidence of chloroquine resistance in tropical Africa. This concern is even greater at the present time as a team from the Division of Insect Borne Diseases of the Medical Research Laboratory, Nairobi, Kenya, visited the Embu area in August 1971, and could not find any evidence of $P$. falciparum resistance to chloroquine.

There is no doubt that the publication of the report by Yoshinaga et al., which was followed by a promotional campaign in favour of the new drug, led to some confusion and uneasiness. It also undermined the confidence of the medical profession and of the population in the therapeutic value of chloroquine, still one of our most reliable and useful antimalarial drugs.

One of the most important duties facing malariologists today is the early and reliable recognition of drug resistance on the basis of field tests. A usable method has been recommended by the W.H.O. ${ }^{1}$ and the strictest scientific criteria should be applied for final assessment of results.-We are, etc.,

\section{J. Bruce-Chwatt}

Ross Institute,

London W.C.

J. M. D. ROBERTS

Medical Research Laboratory,

Nairobi, Kenya

1 World Health Organization. Chemotherapy of Meneva, W.H.O. 1967 .

\section{Endocrine and Metabolic Manifestations of Cancer}

SIR,-Dr. E. J. Ross (18 March, p. 735) took on the difficult task of reviewing the endocrine and metabolic manifestations of cancer. There are some points which I feel need clarification.

In Table I corticotrophin is said to be produced by both papillary and medullary thyroid cancers. To my knowledge the evidence that papillary thyroid carcinoma produces Cushing's syndrome is nonexistent. ${ }^{1}$ Gastric cancers are also listed, but in fact only gastric carcinoids have been proved to cause Cushing's syndrome. The pancreas is listed twice: the first time, I believe correctly, as islet-cell tumours. The second time it is listed as "pancreas" unspecified further, implying, though not stating, that carcinoma of the exocrine pancreas can produce Cushing's syndrome (as yet unproved).

Vasopressin production is said to occur with cerebellar haemangioblastoma: a reference to this would have been appreciated. It is a pity that the well documented pancreatic (probably islet-cell) tumours were not listed. Parathormone production is correctly attributed to bladder, vulval, and other carcinomas, but it is curious that renal carcinoma has been omitted when, by itself, it accounts for over $25 \%$ of reported cases. Ovarian carcinoma also deserves mention in preference to, say, vulval carcinoma. Is precocious puberty really caused by carcinoma of the bronchus? Again, the production of thyroid-stimulating hormone by bronchial carcinoma is so poorly recognized as to deserve a reference.

The lay-out in Table II is very misleading. Section 2 of this is likely to be interpreted as meaning that lymphomas are the tumours most frequently associated with acanthosis nigricans and that ovarian tumours are associated with herpes zoster. The alternative interpretation of this section is almost equally erroneous.

Turning to the end of the review, is it rue that "scleroderma ... developing afte the age of 40," frequently has "a back ground of cancer," unless this refers only to the extremely rare scleroderma-like picture associated with the carcinoid syndrome? Acanthosis nigricans cannot in any sense be termed a "less specific" skin manifestation. It is clinically a distinct entity and associated almost exclusively with very specific types of adenocarcinoma. The lumping together of osteoarthropathy and digital clubbing gives a misleading impression of the way these disorders behave after resection of a lung tumour. The association of arterial thrombosis with renal carcinoma surely calls for a reference.

In relation to patients with bronchial carcinoma, Dr. Ross states that " $2 \%$ of 100 consecutive admissions had adrenal hyperplasia." He appears to have omitted the words "Cushing's syndrome associated with" after the word "had." Adrenal hyperplasia is very common with bronchial carcinom in general. Dr. Ross has unfortunately mis read the Hammersmith Hospital figures: the $6 \%$ incidence of hypercalcaemia in bronchial carcinoma refers to a clinical series and not, as he states, to a necropsy one. The incidence of hypercalcaemia in our series is the same as that found by others who have looked for it. The low incidence of Cushing's syndrome found by us is in agreement with the findings of Kato et al..$^{2}$; the latter is I believe the only paper which gives the incidence of Cushing's syndrome in a very large unselected series of oat-cell carcinomas.

I am afraid that the review of this important subject has been spoilt by some factual errors, misleading impressions, and important omissions.-I am, etc.,

\section{J. G. Azzopard}

Department of Pathology,

Royal Postgraduate Medical School,

London W.12 1 Azzopardi, J. G., and Williams, E. D., Cancer
(Philadelphia), 1968, 22, 274.
2 Kato, Y., Ferguson, T. B., Bennett, D. E., and
Burford, T. H., Cancer (Philadelphia), 1969,
23, 517.

SIR,-It is not clear why Dr. E. J. Ross (18 March, p. 735) included gastrin secreting islet-cell tumours of the pancreas (D-cell, and no longer $\alpha$-cell) in his list of hormones secreted by malignant cells and their sites of origin. A gastrinoma which can produce the Zollinger-Ellison syndrome is comparable to an insulinoma in which the production of insulin, Dr. Ross agrees, is not "ectopic." He might equally well have included calcitonin production by a medullary carcinoma of the thyroid gland, and so on.

The article by Dr. Ross was about the secretion of substances with hormonal actions (indistinguishable from hormones secreted by normal endocrine tissues) by tumours derived from tissues that do not normally secrete hormones. The production of these substances has been called "ectopic" or "inappropriate," but what is wanted is a better name than either of these two. Any tumour of endocrine tissue which secretes a hormone does so inappropriately to give in time the relevant syndrome of overproduction, and any metastasis of such a tumour which also secretes the hormone is "ectopic." Sometimes endocrine tissue may be ectopic, that is separate from a normal sited endocrine organ, and occasionally ectopic tissue may be the only source of a hormone (with agenesis of the endocrine organ in its normal site). It is probable that endocrine cells producing one polypeptide hormone are capable of producing other ones and sometimes do. Is this to be called "inappropriate" or "ectopic"? The naming of the hormonal secretions from "non-endocrine" tumours needs to be more accurate than "ectopic" and "inappropriate," but it is difficult to see what it should be. Perhaps "non-endocrine hormone production" which is purposely rather vague might be acceptable even if not very satisfactory.

Many of the substances secreted by nonendocrine tumours which can show hormona actions are identical to normal hormones on all tests to which they have been submitted. These tumours are usually, however, composed of relatively dedifferentiated cells unlike the cells of endocrine tumours which are mostly relatively well-differentiated. It is important to make certain of the origin of overproduction of a hormone, because if it comes from a malignant tumour of endocrine tissue the prognosis is often good so long as the effects of the hormonal oversecretion can be moderated. A malignant insulinoma or carcinoma of a parathyroid gland is an instance of this. And a gastrinoma is another example if a total gastrectomy has been performed. It is for this reason that the inclusion of this tumour in the list in the paper by Dr. Ross is best avoided.-I am, etc.,

MiLO KeYNES

Oxford

\section{Diagnosis of Potassium Chloride Stricture}

SIR,-In "Second Opinion, Please" (25 March, p. 797) Dr. Brian R. Wilkinson and Mr. John Alexander Williams tell the long and fascinating story of delay in the diagnosis of a Salupres stricture. As the patient progressed through biochemical profiles, barium studies, and scintillation scans and then hovered on the brink of arteriography, the heart of every tax-paying reader must have sunk a little.

It is perhaps pertinent to recall the diagnosis of one of the first Salupres strictures which I presented to the Royal Society of Medicine in 1966. The patient was under the care of Mr. Andrew Desmond at St. James's Hospital, Balham. The pain was similarly vague and non-specific and his barium meal was normal. However, the erect abdominal plain film showed a few definite fluid levels in the small intestine and this led to laparotomy, at which a typical potassium stricture was excised.

I believe there are two important morals which should have been drawn from this case. Firstly, any patient with vague abdominal colic of this kind should have an erect and supine plain film performed at an 